\title{
Bases d'un modèle décrivant la régulation du métabolisme du glucose de la chèvre en lactation
}

\author{
D Sauvant ${ }^{1}$, J Grizard ${ }^{2}$ \\ 1 INRA, station de nutrition et alimentation, Institut national agronomique Paris-Grignon, \\ 16, rue Claude-Bernard, 75231 Paris Cedex 05; \\ 2 INRA, centre de recherches de Clermont-Ferrand, laboratoire du métabolisme azoté, \\ 63122 Ceyrat, France
}

\begin{abstract}
Summary - Basis of a model describing the regulation of glucose metabolism in the lactating goat. A model with 5 compartments, 1 for glucose, 2 for insulin and glucagon, was elaborated to simulate the glucose metabolism of the dairy goat. Validation was carried out from data of short-term response to injection of both hormones.
\end{abstract}

Introduction - Les résultats des recherches foralisées sur le métabolisme du glucose du ruminant, à notre connaissance, n'ont pas été utilisés pour mettre en place un modèle dynamique permettant de traduire ses variations à court terme.

Matériels et méthodes - Le système, conçu pour être le plus simple possible, comprend 5 compartiments: le glucose du sang (GLO), un compartiment d'accumulation et un d'échange et d'élimination, pour le glucagon $(\mathrm{G} 1, \mathrm{G} 2)$ et l'insuline $(11,12)$ respectivement. Le compartiment GLO est alimenté par ses précurseurs issus du tube digestif, transformés en glucose par des réactions obéissant à la loi d'action de masse, et par le flux endogène issu de la néoglucogénèse (NGG). Sa modélisation (tableau I) a été faite par la combinaison de PGLOG, la NGG activée par la concentration en glucagon (CGLA), et de PGLOI, la NGG limitée par la concentration en insuline (CINS). Les 3 flux d'utilisation du glucose ont été consacrés à l'entretien, supposé constant, la mamelle $(1,3 \times$ lactose produit) et la captation insulino-dépendante par les tissus périphériques non mammaires (UTIS). Les com jartiments $\mathrm{G} 1$ et 11 sont alimentés par les sécrétions de ces hormones (SINS, SGLA) qui sont respectivement activées et inhibées par un accroissement de la glycémie (CGLO).

Les variations de ce système ont été décrites par 5 équations différentielles dynamiques du $1^{\text {er }}$ ordre (Jolivet, 1983), la simulation procède selon la méthode d'Euler avec un pas de temps d' 1 min. Les régulations de l'intensité (I) des voies métaboliques sont exprimées par la loi de Michaelis-Menten en fonction de la concentration en substrat régulateur $(s): 1=\operatorname{IMAX}\left(1+(k / s)^{n}\right)^{-1}, k$ représente la constante de Michaelis et le signe de l'exposant $n$ permet de traduire un phénomène d'activation (+) ou d'inhibition (-), en outre ses valeurs supérieures à 1 permettent d'exprimer des relations sigmoïdales (tableau 1). Les valeurs des constantes d'échange et d'élimination entre les compartiments G1 et G2, d'une part, et I1 et 12 d'autre part, ainsi que celles des lois de régulation des flux PGLOI, PGLOG, UTIS (tableau I), ont été calculées à partir de résultats de tests d'injection réalisés au laboratoire du métabolisme azoté de I'INRA-Theix (Debras et al, 1988). L'identification des lois a été conduite par itération de la procédure de validation (tableau 1).

La validation du modèle a été conduite sur les réponses à des injections à dose unique de glucagon ou d'insuline, dans les compartiments $\mathrm{G} 1$ et $\mathrm{I1}$, à des chèvres de $60 \mathrm{~kg}$ de poids vif produisant $3 \mathrm{~kg}$ de lait/j et ingérant $1.4 \mathrm{~kg}$ de matière organique digestible/j.

Résultats et discussion - En raison de l'absence de données expérimentales, la loi de hiérarchie des effets des 2 hormones sur la NGG, a été déterminée de façon théorique en fonction du rapport CINS/CGLA. II a par ailleurs été nécessaire d'introduire un processus d'homéostase pour éviter que la glycémie simulée ne devienne négative après l'injection d'insuline. 
Tableau I. Valeurs des paramètres des lois de régulation.

\begin{tabular}{|c|c|c|c|c|}
\hline $\begin{array}{c}\text { Voie } \\
\text { metabolique } \\
\text { (I) }\end{array}$ & $\begin{array}{l}\text { Paramètre } \\
\text { régulateur }\end{array}$ & $\begin{array}{c}\text { Intensité } \\
\text { maximale } \\
(I M A X)\end{array}$ & $\underset{(\mathrm{n})}{\text { Exposant }}$ & $\begin{array}{l}\text { Constante } \\
\text { de Michaelis } \\
\text { (k) }\end{array}$ \\
\hline $\begin{array}{l}\text { PGLOI } \\
\text { PGOLG } \\
\text { UTIS } \\
\text { SINS } \\
\text { SGLA }\end{array}$ & $\begin{array}{l}\text { CINS } \\
\text { CGLA } \\
\text { CINS } \\
\text { CGLO } \\
\text { CGLO-200 }\end{array}$ & $\begin{aligned} 200 \mathrm{mg} / \mathrm{mn} \\
275 \mathrm{mg} / \mathrm{mn} \\
170 \mathrm{mg} / \mathrm{mn} \\
2500 \mathrm{ng} / \mathrm{mn} \\
500 \mathrm{ng} / \mathrm{mn}\end{aligned}$ & $\begin{array}{l}-2 \\
+1 \\
+3 \\
+3 \\
-1\end{array}$ & $\begin{array}{rr}1,5 & \mathrm{ng} / \mathrm{ml} \\
0,1 & \mathrm{ng} / \mathrm{ml} \\
3 & \mathrm{ng} / \mathrm{ml} \\
800 & \mathrm{mg} / \mathrm{l} \\
65 & \mathrm{mg} / \mathrm{l}\end{array}$ \\
\hline
\end{tabular}

Les figures 1 et 2 indiquent les valeurs mesurées et simulées de CGLO et CINS après une injection de $520 \mu \mathrm{g}$ de glucagon et de la glycémie après une injection de $681 \mathrm{\mu g}$ d'insuline (Grizard et al, 1988). Les amplitudes des réponses sont globalement respectées, néanmoins les valeurs simulées après injection de glucagon présentent un retard de l'ordre de 10 à $20 \mathrm{~min}$, alors qu'une tendance inverse apparaît après l'injection d'insuline.

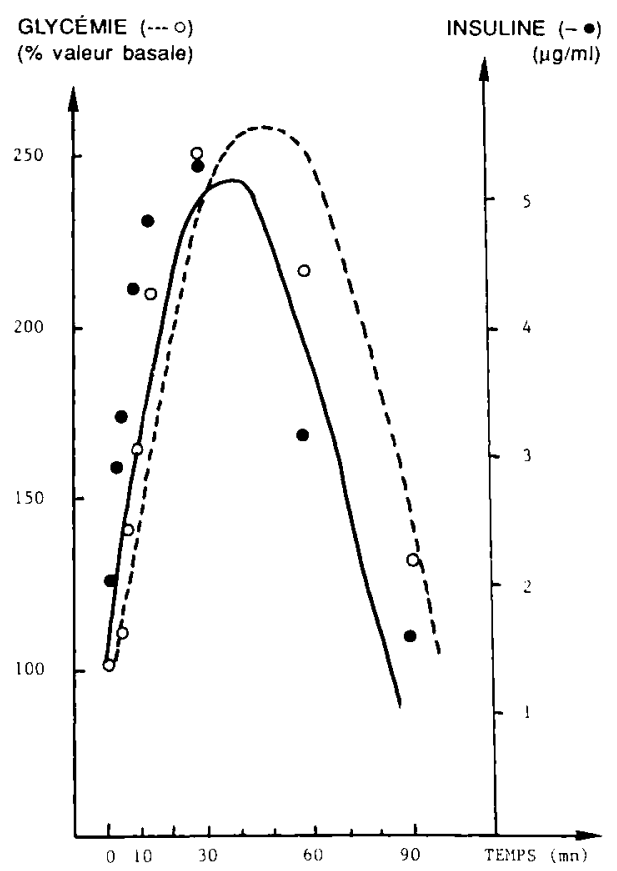

Fig 1. Variations mesurées et simulées de la glycémie et de l'insulinémie après une injection de $520 \mu \mathrm{g}$ de glucagon.

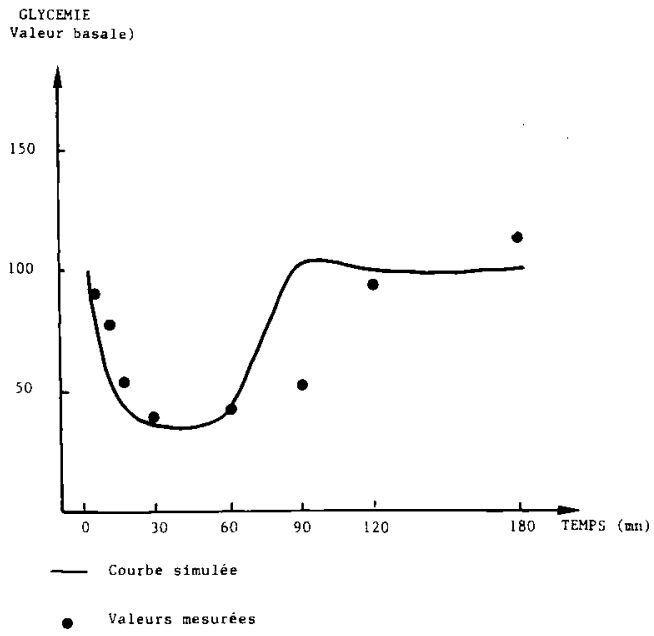

Fig 2. Variations mesurées et simulées de la glycémie après une injection de $681 \mu \mathrm{g}$ d'insuline.

Ces résultats ont permis de souligner la nécessité de préciser l'organisation des actions des 2 hormones vis-à-vis de la néoglucogénèse; par ailleurs, certaines caractéristiques dynamiques de ce modèle demandent à être améliorées, vraisemblablement aux dépens de sa simplicité.

Debras E, Grizard J, Aina E, Tisserand S, Champredon C, Arnal M (1988) Am J Physiol 256, E295-E302

Grizard J, Champredon C, Aina E, Sornet C, Debras E (1988) Horm Metab Res 20, 71 76

Jolivet $E$ (1983) Introduction aux modèles mathématiques en biologie. Masson, Paris 